\title{
Construção de um kit experimental com arduino para ensino de oscilações em tempo real
}

Experimental kit for studing real-time oscilations using arduino

\author{
Pedro Davi Matos Pereira ${ }^{* 1,2 @}$, Marcelo Souza da Silva ${ }^{2}$ \\ ${ }^{1}$ Instituto de Física, Universidade Federal da Bahia, Campus Universitário de Ondina, Salvador, BA, Brasil. \\ ${ }^{2}$ Instituto Federal de Educação, Ciência e Tecnologia do Sertão Pernambucano, Grupo de Pesquisa em Ensino de Ciências, \\ Salgueiro, PE, Brasil.
}

Recebido em 18 de maio de 2021. Revisado em 06 de julho de 2021. Aceito em 26 de agosto de 2021.

\begin{abstract}
O estudo das oscilações pode se configurar como uma rica oportunidade para o desenvolvimento de atividades experimentais. Entretanto, devido a uma série de dificuldades instrumentais, muitos professores optam por trabalhar apenas com um cronometro para obtenção da frequência média das oscilações. A aquisição dos dados de posição em função do tempo está disponível, em geral, para quem tem acesso a kits experimentais caros. Neste artigo propomos a construção e utilização de um kit experimental para estudo de oscilações utilizando arduino. Por meio de um sensor ultrassônico HC-SR04, pode-se construir gráficos de posição (velocidade e aceleração) em tempo real, permitindo a visualização do comportamento senoidal apresentado pela teoria, e possibilitando maior compreensão do fenômeno além da determinação de grandezas físicas envolvidas. O kit inclui um software desenvolvido em $\mathrm{C}++$, denominado PMOscillator, responsável pela aquisição, processamento, plotagem em tempo real e análise dos dados.
\end{abstract}

Palavras-chave: Arduino, oscilações, kit didático.

\begin{abstract}
The study of oscillations can be configured as a rich opportunity for the development of experimental activities. However, due to a series of instrumental difficulties, many teachers choose to work only with a stopwatch to obtain the average frequency of the oscillations. To obtain from the data of position versus time is generally available for those who have access to expensive experimental kits. In this article we propose the construction and use of an experimental kit for study of oscillations using arduino, as well as a proposal of use. With a ultrassonic sensor HC-SR04, we can construct real position graphs, allowing a visualization of the sinusoidal behavior visualized in the theory, and also enabling a greater understanding of the phenomenon and the determination of the physical quantities involved. The kit includes a software developed in $\mathrm{C}++$, called PMOscillator, responsible for the acquisition, processing, real-time plotting and data analysis.
\end{abstract}

Keywords: Arduino, oscilations, didatic instrumentation.

\section{Introdução}

O desenvolvimento de atividades experimentais educativas que possam exemplificar a intima relação entre os aspectos teóricos e experimentais que permeiam a construção do saber científico pode fomentar um ambiente de investigação que desperte a atenção e curiosidade dos alunos acerca do tema em questão. Quando se trata do estudo de oscilações mecânicas, no geral, adotam-se práticas experimentais baseadas na medição do período e da amplitude do sistema com os instrumentos régua e cronômetro. Embora esse tipo de estudo seja eficiente para verificar o comportamento periódico do movimento, não consegue explicitar o comportamento senoidal esperado para o gráfico da posição em função do tempo, devido à dificuldade na medição simultânea destas duas grandezas utilizando os instrumentos mencionados. Neste cenário, a necessidade

\footnotetext{
*Endereço de correspondência: pedrophysicist@gmail.com
}

de propor uma instrumentação acessível, e que permita a obtenção de dados mais fidedignos do sistema em estudo, podem tornar o estudo do tema mais estimulante. Algumas abordagens presentes na literatura utilizam ferramentas de videoanálise, como o Tracker [1]. Uma outra alternativa para isto é a utilização de ferramentas iterativas, como experiências assistidas pelo PC [2]. Uma importante ferramenta nesta proposta é o arduino, placa de prototipagem eletrônica open source de baixo custo, fácil utilização e implementação [3]. Ela é amplamente utilizado em diversas áreas quando há a necessidade da elaboração de um dispositivo eletrônico; a exemplo, para o desenvolvimento de um sensor barato para monitoramento de líquidos [4, e a construção de um audiotermômetro para inclusão de estudantes cegos [5]. Também encontra-se na literatura trabalhos na área de física e ensino de física, tendo como exemplo a construção de um dispositivo barato para análise do movimento de magnetos em tubos verticalmente orientados [6], onde se estudou a queda retardada do imã no tubo condutor 
com o sensor ultrassônico HC-SR04, e um dispositivo para construção de gráficos de movimento em tempo real [7, onde se utilizou o arduino em conjunto com um sensor ultrassônico HC-SR04 e o software PLXDAQ, que agrupa os dados recebidos numa planilha do Microsoft Excel para que depois possam ser plotados. No estudo de oscilações, mais uma vez, também é possível encontrar a aplicação do arduino, como a construção de instrumentação para análise de vibração no espaço da posição e das frequências estudando-se a oscilação de uma haste metálica com acelerômetro e giroscópio acoplados a ela [8]. Contudo, quando utilizado para fins didáticos, na maioria das vezes os trabalhos são na área de eletrônica ou, na física, para eletricidade ou física moderna 9 11.

\section{O Kit Didático}

O kit proposto visa tornar o ensino de oscilações por meio da experimentação mais lúdico, permitindo relacionar o fenômeno de forma mais evidente com a teoria através da construção em tempo real de gráficos da posição em função do tempo de forma totalmente interativa.

O kit didático é pode ser subdivido em quatro partes:

(i) Um módulo medidor de distância;

(ii) Software PMOscillator, responsável pela aquisição, por armazenar, plotar e analisar os dados medidos pelo módulo;

(iii) Osciladores nos tipos pêndulo e massa-mola;

(iv) Suportes para o oscilador e detector.

\subsection{Medidor de distância}

O módulo medidor de distância, mostrado na Figura 1 é a parte do kit capaz de verificar a posição do corpo oscilante em tempo real. Para isto, foi utilizado um sensor ultrassônico HC-SR04 ligado a um Arduino modelo Nano v3.0 [12], como ilustrado na Figura 2 Ambos componentes foram armazenados numa caixa de montagem na cor preta, adaptada para o posicionamento do sensor e saída do cabo USB.

O HC-SR04 é um sensor de distância do tipo ultrassônico capaz de medir distâncias de $3 \mathrm{~cm}$ a $4 \mathrm{~m}$ com precisão de $3 \mathrm{~mm}$ [13. O módulo possui um conjunto emissorreceptor de ondas sonoras na frequência de $40 \mathrm{kHz}$ (ultrassom). Quando acionado, através de um sinal de $10 \mathrm{~ms}$ na porta Trigger, o dispositivo emite 8 pulsos ultrassônicos que refletirão no anteparo e retornarão ao detetor. O tempo entre a emissão e detecção é registrado e, conhecendo-se velocidade do som, a distância poderá ser determinada através da relação

$$
d=\frac{1}{2} v_{s} t
$$

em que o $d$ é a distância do sensor ao anteparo, $v_{s}$ é a velocidade do som no ar e $t$ é o tempo referente a ida e

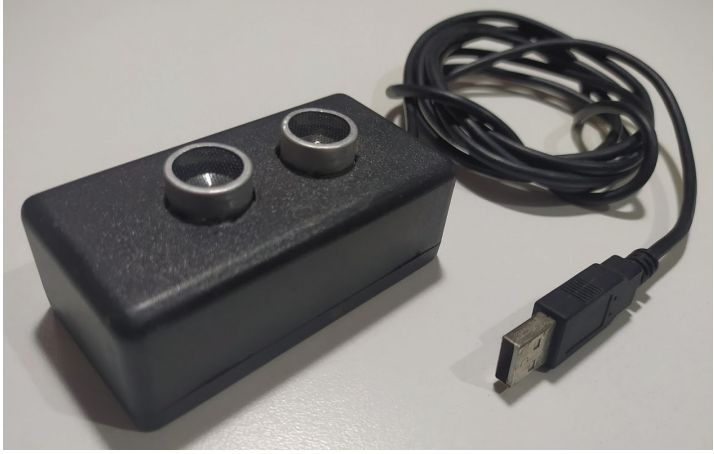

Figura 1: Foto do módulo detetor de distância montado.

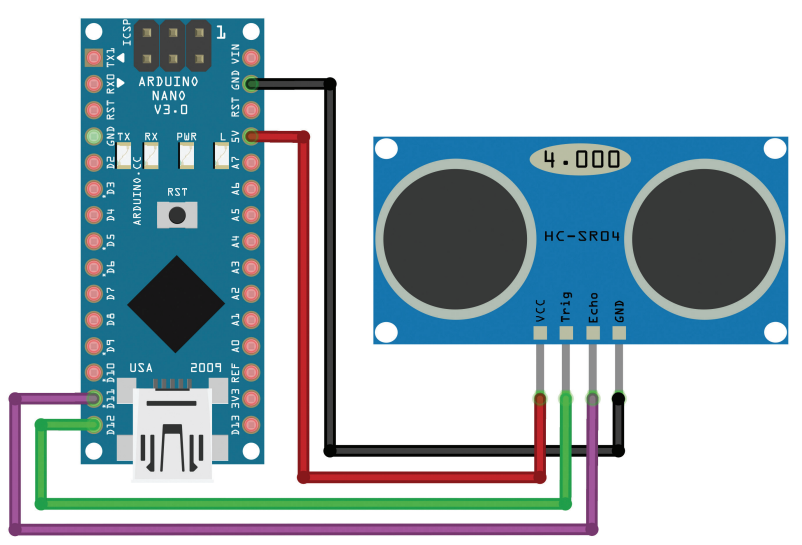

fritzing

Figura 2: Arduino nano conectado so sensor HC-SR04.

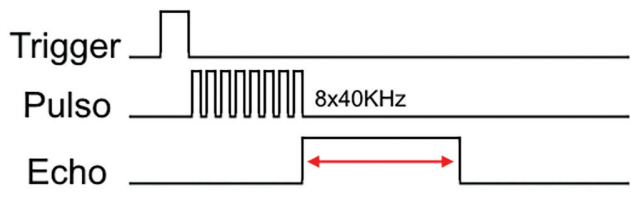

Figura 3: Diagrama de tempo do HC-SR04.

volta do pulso, que deverá ser compensado pelo termo $1 / 2$ presente na expressão. O processo descrito é melhor representado pelo diagrama da Figura 3

O arduino é o componente do medidor capaz de acionar o sensor a cada $0,1 \mathrm{~s}$ e verificar o tempo de deslocamento do pulso ultrassônico. O valor registrado é enviado via comunicação serial [14] através do cabo USB conectado a ele, que também é responsável por alimentar todo o circuito. O código programado na placa pode ser encontrado no apêndice.

Não obstante, o sensor ultrassônico HC-SR04 utilizado para medir distâncias possui abertura de campo de aproximadamente $15^{\circ}$ para direita e para esquerda, como informado em seu datasheet [13, e representado na Figura 4 e na Figura 5 Isto significa que, a depender da distância e das dimensões do anteparo, outros objetos na 


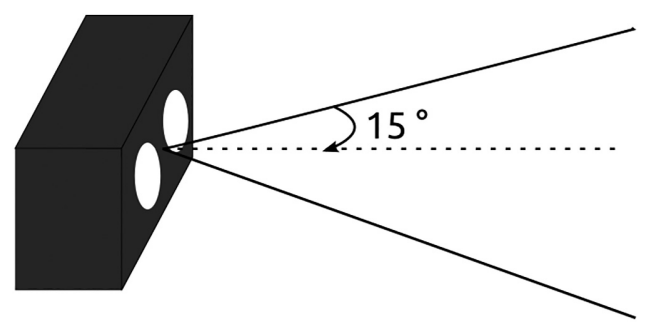

Figura 4: Representação da região do ângulo de abertura do sensor HC-SR04.

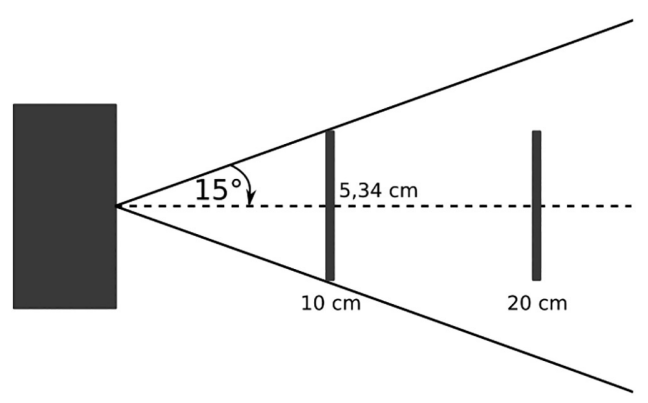

Figura 5: Visão superior da região de aquisição do sensor HCSR04, com anteparos posicionados a $10 \mathrm{~cm}$ e $20 \mathrm{~cm}$ de distância.

abertura posicionados na região de alcance do detector poderão interferir nas medidas, reduzindo a precisão na aquisição dos dados. De acordo com Dworakowski et al. 7], na prática a região que pode causar interferência é ainda menor, mas para garantir qualidade na produção e coleta de dados é aconselhável seguir as recomendações do fabricante.

\subsection{Software}

As informações adquiridas pelo módulo medidor de distância deverão ser processadas e armazenadas pelo computador através de um software externo ao circuito. Para isto, foi desenvolvido um software em $\mathrm{C}++$, denominado PMOscillator, com interface gráfica interativa e de fácil manuseio totalmente dedicado ao kit, como apresentado na Figura 6 O professor poderá obtê-lo no repositório indicado na referência [15]. O software oferece ferramentas de análise dos dados aferidos, relacionandoos à teoria de oscilações, mas também pode ser utilizado para plotar a posição em função do tempo para outros experimentos em geral.

\subsubsection{Aquisição e processamento}

Para utilizar o kit, basta conectar o módulo medidor de distância ao computador via USB que o PMOscillator o reconhece automaticamente. $\mathrm{O}$ software recebe do arduino o intervalo de tempo, em milissegundos, que um dos pulsos emitidos leva entre a saída e o retorno ao receptor. Esta informação é processada, de forma a transformá-la na distância do corpo até o sensor, através da aplicação da relação (1).

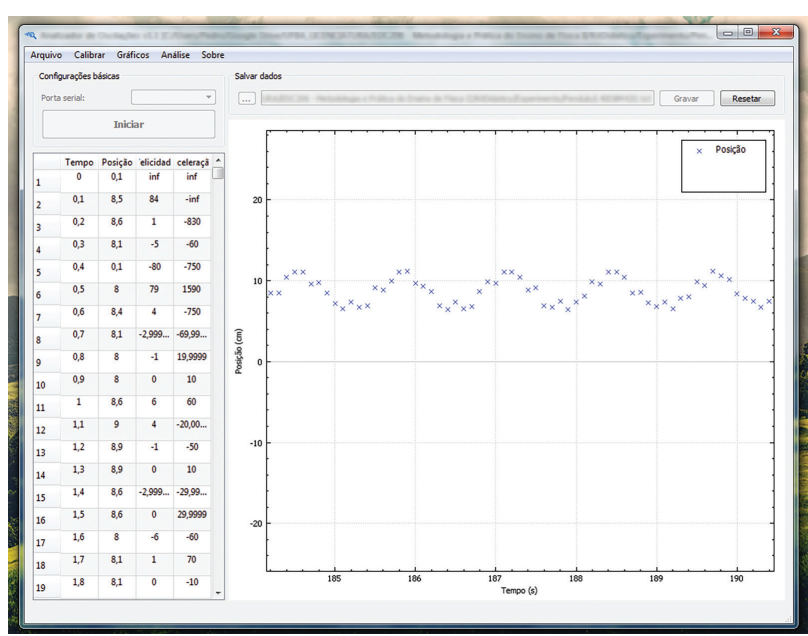

Figura 6: Gráfico de posição plotado em tempo real.

Para isto, uma informação necessária é a velocidade do som no ar. O software estabelece como valor padrão $340 \mathrm{~m} / \mathrm{s}$, que pode ser alterado através da opção de calibração. Posicionando-se um anteparo a uma distância conhecida, afere-se o tempo de deslocamento do pulso e, com isso, a velocidade do som é determinada.

\subsubsection{Análise}

O software também possui uma ferramenta de análise, mostrada na Figura 7 capaz de determinar a equação horária que melhor descreve um conjunto de pontos. Tomando como referência a curva senoidal

$$
y=A \sin (B x+C)+D
$$

o método consiste em encontrar os valores dos parâme$\operatorname{tros} A, B, C$ e $D$ que minimizem a função

$$
\delta=\sum_{i=0}^{N}\left|y\left(x_{i}\right)-y_{i}\right|^{2}
$$

em que $x_{i}$ e $y_{i}$ são as coordenadas do i-ésimo ponto dentre os $N$ compreendido no intervalo tomado para análise.

Para minimização, foi utilizado um o método de Levenberg-Marquardt [16 18, utilizando-se o algoritmo implementado na biblioteca GNU Scientific Library [19].

\subsection{Osciladores}

Como objetos de estudo, o kit permite, a princípio 1 o estudo de osciladores do tipo pêndulo simples e massamola vertical. Ele permitirá determinar a posição do pêndulo ou da massa em função do tempo, e analisar os dados obtidos.

\footnotetext{
1 O kit pode ser facilmente adaptado para estudo de outros sistemas. A título de exemplo, o pêndulo amortecido.
} 


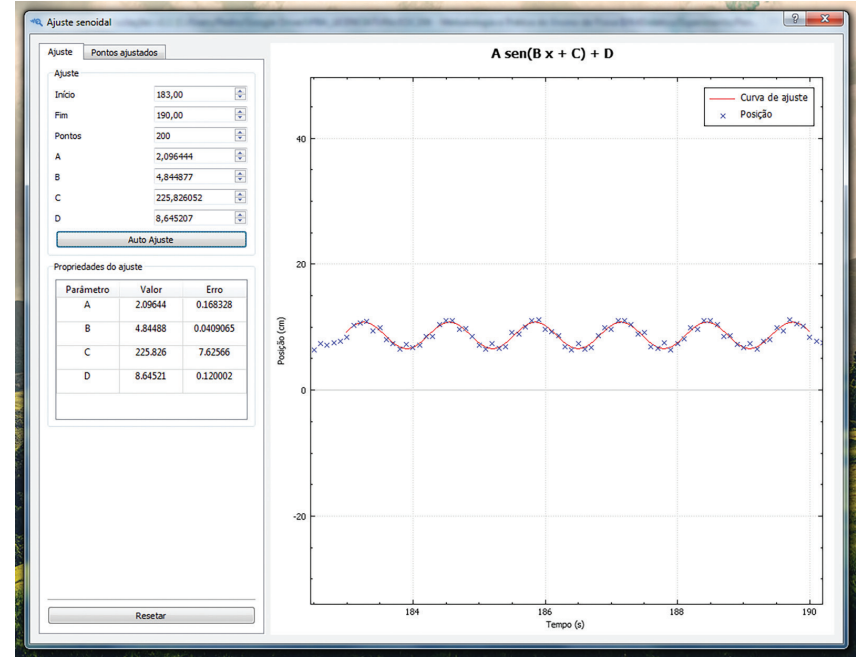

Figura 7: Valores dos parâmetros e seus respectivos erros na curva ajustada (em vermelho) no intervalo de tempo especificado pelo usuário.

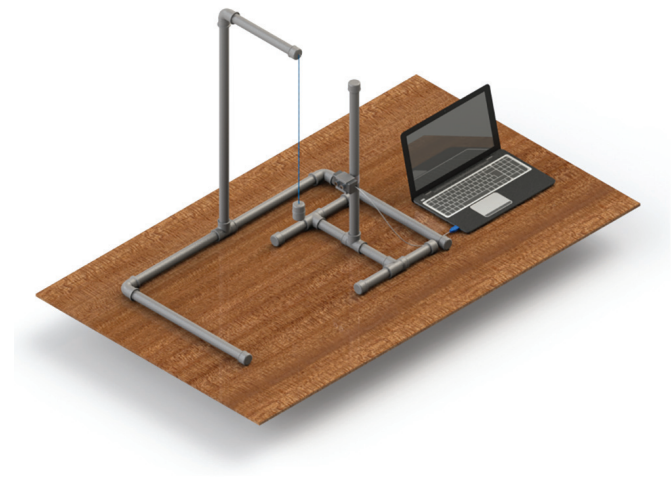

Figura 8: Montagem para estudo do oscilador tipo pêndulo simples.

\subsubsection{Pêndulo simples}

Para montagem do pêndulo, utiliza-se o suporte, massas e cordão inextensível, além do detetor de distâncias. O suporte oferece a possibilidade de pêndulos com fio de até $140 \mathrm{~cm}$ de comprimento. As massas contidas no kit foram construídas cada uma com duas unidades de cap PVC de $40 \mathrm{~mm}$ de diâmetro preenchidos com pesos de pesca; a menor massa possui $100 \mathrm{~g}$ e a maior, $420 \mathrm{~g}$. As dimensões finais das massas foram $6 \mathrm{~cm}$ de altura e $4 \mathrm{~cm}$ de largura. A construção de massas nestas dimensões facilita a aquisição do detetor. Como a distribuição dos pesos de pesca não é necessariamente uniforme, é necessário considerar a localização do centro de massa nos resultados obtidos.

A Figura 8 ilustra o aspecto final da montagem. Note que é necessário a utilização de um suporte auxiliar para o módulo detetor de distâncias, pois é necessário estar no nível das oscilações para que as medidas sejam realizadas corretamente.

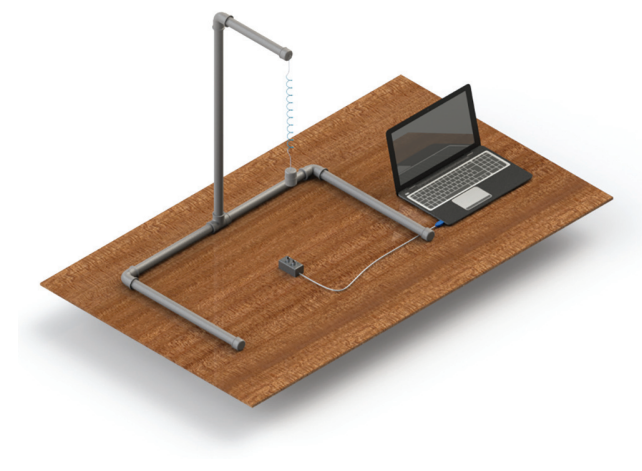

Figura 9: Montagem para estudo do oscilador massa-mola. $O$ suporte para o sensor não é necessário neste caso.

\subsubsection{Massa-mola vertical}

Para montagem do massa-mola vertical, utiliza-se o suporte, massas e molas, além do detetor de distâncias. Os pesos utilizados são os mesmos do pêndulo. A Figura 9 ilustra o aspecto final da montagem. Note que neste caso a altura do suporte deve se adequar ao comprimento da mola distendida, uma vez não há ajuste de altura no posicionamento do detetor. Este é uma sugestão para testes iniciais, mas o suporte pode ser adaptado para outras configurações.

\subsection{Suportes}

Os suportes foram feitos com tubos de PVC, devido ao baixo custo e facilidade de montagem. Além disto, a portabilidade oferecida pela utilização dos canos facilita o manuseio e transporte do kit pelo professor. O que seria diferente no caso da utilização de suportes feitos de alumínio, madeira ou ferro.

\section{Proposta de Experimento}

Da teoria, sabe-se que a função horária da posição relacionada ao movimento tanto do pêndulo quanto do oscilador massa mola possui comportamento senoidal. Ou seja,

$$
x(t)=A \sin \left(\omega t+\varphi_{0}\right),
$$

em que $A$ é a amplitude da oscilação, $\varphi_{0}$ é a fase inicial, relacionada à posição inicial do corpo, e $\omega$ é a frequência angular.

Tanto a amplitude quanto a posição são parâmetros de ajuste do sistema. Contudo, $\omega$ é intrínseco às características físicas do mesmo. No caso do pêndulo, ele se relaciona com o comprimento do fio e a aceleração gravitacional, podendo ser escrito como

$$
\omega=\sqrt{\frac{g}{L}}
$$

ou, no caso do oscilador massa mola, ele se relaciona com a massa e a constante elástica da mola utilizada, dada 
pela expressão

$$
\omega=\sqrt{\frac{K}{m}} .
$$

Com o kit é possível encontrar a função horária da posição, em tempo real, no formato

$$
x(t)=A \sin (B * t+C)+D .
$$

Por comparação direta com (4), podemos relacionar os parâmetros do ajuste às grandezas físicas do sistema. Em especial, o parâmetro $B$, que corresponde à frequência angular, e o parâmetro $D$, relacionado à distância do ponto de equilíbrio do corpo até o sensor.

Com isto, pode-se variar as grandezas físicas envolvidas e investigar o comportamento da função horária, bem como período de oscilação. Para isto, o professor terá a autonomia de escolher uma teoria do laboratório didático para aplicar com seus alunos.

\section{Resultados do Experimento}

A precisão do sensor ultrassônico HC-SR04 de até $3 \mathrm{~mm}$ oferece a possibilidade de obtenção de valores bem próximos da realidade. Por exemplo, realizando-se o experimento do pêndulo com comprimento do fio de $40 \mathrm{~cm}$, ajustando uma amplitude de $2 \mathrm{~cm}$ e posicionando a uma distância inicial de $8,5 \mathrm{~cm}$ obtivemos, através do ajuste não linear, os seguintes valores para as constantes:

$$
\begin{aligned}
& A=(2,0 \pm 0,2) \mathrm{cm}, \\
& B=(4,84 \pm 0,04) \mathrm{s}^{-1}, \\
& C=(225,8 \pm 7,6), \\
& D=(8,6 \pm 0,1) \mathrm{cm} .
\end{aligned}
$$

como mostrado na Figura 7] Os desvios associados são os erros estimados na regressão, que também são fornecidos pelo software. Os pontos obtidos na realização deste experimento apresentam baixo resíduo em relação à curva ajustada. A qualidade desta medida está associada à dimensão do oscilador, cujo diâmetro é próximo de $4 \mathrm{~cm}$, ocupando quase toda a abertura de $4,5 \mathrm{~cm}$ do detetor à distância de $8,5 \mathrm{~cm}$.

Note que a amplitude obtida neste caso é igual à que foi utilizada na configuração inicial e a distância $D$ da posição de equilíbrio até o sensor foi determinada com discrepância de 1\%. Com efeito, a equação (4) com os parâmetros ajustados pode ser expressa por

$$
x(t)=2,0 \sin (4,84 t+225,8)+8,6 .
$$

Sendo $B$ a frequência angular, representada por $\omega$ na expressão (5), podemos determinar o comprimento do pêndulo com base nos dados experimentais. Para isto, é necessário conhecer o valor da aceleração gravitacional no local $g$.
Para efeito de comparação, o valor da gravidade no local pode ser obtida por vias tradicionais utilizando o próprio pêndulo e um cronômetro. Uma forma precisa de fazer isto é aferir a frequência de oscilação através da medição do intervalo de tempo sobre 10 oscilações, por exemplo, e a repetição estatística da medida. Com esta, determina-se $g$ utilizando-se a expressão (5).

Ao realizar este experimento, com um pêndulo de comprimento $L=(1,000 \pm 0,002) m$, com período de uma oscilação $T=(2,004 \pm 0,006)$, obtivemos como resultado

$$
g=(9,830 \pm 0,062) \mathrm{m} / \mathrm{s}^{2}
$$

Este resultado está de acordo com valores obtidos em trabalho recente explorando diferentes métodos [20].

Utilizando-se o valor de $B$ ajustado com sua incerteza estimada, considerando o valor da aceleração gravitacional determinado e propagando as devidas incertezas [21], pode-se chegar ao resultado

$$
L^{\prime}=(0,42 \pm 0,01) m,
$$

que possui discrepância de apenas $5 \%$ do comprimento do fio utilizado. Por outro lado, levando em conta as dimensões do corpo oscilante e considerando que o centro de massa esteja aproximadamente no centro geométrico, a expectativa teórica é $L \approx 43 \mathrm{~cm}$, o que torna a discrepância próxima de $2 \%$.

O experimento apresenta excelente reprodutibilidade. Além disso, a depender da forma como o aluno realiza o experimento e a obtenção dos dados, é possível obter resultados ainda mais acurados. É importante ressaltar que a qualidade das medidas está diretamente ligada ao conjunto de pontos analisados, e o desenvolvimento da perspicácia investigativa envolvida na produção dos dados é uma das habilidades científicas que podem ser aprimoradas na execução de atividades como a que se propõe aqui.

\section{Conclusões}

Possibilitar que professores e estudantes que convivem com as mais diversas condições estruturais em laboratórios didáticos tenham acesso a um experimento que forneça dados com boa fidedignidade, que representem bem o fenômeno em estudo por um baixo custo, é um grande desafio para a área de instrumentação para o ensino de física. Neste artigo propomos a construção e utilização de um kit experimental utilizando arduino e disponibilizamos o software PMOscillator dedicado ao kit, possibilitando a plotagem em tempo real e análise dos dados. Este barato dispositivo permite, por meio de um sensor ultrassônico HC-SR04, construir gráficos de posição (velocidade e aceleração) em tempo real. A visualização do comportamento senoidal constatado pode ser comparado com a modelagem apresentada pela teoria, ou até mesmo determinado somente pela experimentação. Um outro aspecto que pode ser viabilizado 
com a utilização do kit proposto, é que o estudante pode participar da montagem do protótipo para aquisição de dados, conforme o processo de familiarização com as novas tecnologias ocorram, podem também surgir adaptações, novas propostas de investigações, características da apropriação do saber.

\section{Agradecimentos}

A Karla Pedroza Oliveira pela leitura crítica. Aos avaliadores pelas considerações realizadas que muito contribuíram para a melhoria de nosso trabalho. Ao programa PIBID - subprojeto de Física do IFSertãoPE, pelo fomento. À Coordenação de Aperfeiçoamento de Pessoal de Nível Superior (CAPES), pelo apoio financeiro.

\section{Material suplementar}

O seguinte material suplementar está disponível: Apêndice.

\section{Referências}

[1] D. Brown, Tracker: Free Video Analysis and Modeling Tool for Physics Education, https://physlets.org /tracker/

[2] A.R. Souza, A.C. Paixão, D.D. Uzêda, M.A. Dias, S. Duarte e H.S. Amorim, Revista Brasileira de Ensino de Física 33, 1702 (2011)

[3] Página Oficial do Arduíno, disponível em: https://ww w.arduino.cc

[4] D.K. Fisher e R. Sui, IGR J. Agr. Eng. Int. 15, 328 (2013).

[5] H.P. Cordova, C.E. Aguiar, H.S. Amorim, K.S.O.M Sathler e A.C.F. Santos, Revista Brasileira de Ensino de Física 40, e2505 (2018).

[6] R.M. Szmoski, A. Doff, V.M. Lenart, S.K. Schwiderke e L.V.G Fachini, Caderno Brasileiro de Ensino de Física 40, e1505 (2017).

[7] L.A. Dworakowski, A.M. Hartmann, E.M. Kakuno e P.F.T Dorneles, Revista Brasileira de Ensino de Física 38, 33503 (2016).

[8] M. Varanis, A.L. Silva, P.H.A. Brunetto e R.F. Gregolin, Revista Brasileira de Ensino de Física 38, 1301 (2016).

[9] S. Silveira, Desenvolvimento de um kit experimental com Arduino para o ensino de física moderna no ensino médio. Dissertação de Mestrado, Universidade Federal de Santa Catarina, Araranguá (2016).

[10] S. Silveira e M. Girardi, Revista Brasileira de Ensino de Física 39, e4502 (2017).

[11] C.J.M. Souza e M.S. Silva, LINKSCIENCEPLACEInterdisciplinary Scientific Journal 3, 82 (2016).

[12] A. Nano, disponível em: https://store.arduino.cc/ardui no-nano, acessado em 16/05/2021.

[13] Datasheet do HC-SR04, disponível em: http://elecfrea ks.com/estore/download/EF03085-HC-SR04_Ultraso nic_Module_User_Guide.pdf

[14] Especificações da comunicação serial, disponível em: http://i2c.info/i2c-bus-specification acessado em $16 / 05 / 2021$.
[15] Repositório do software PMOscillator, disponível em ht tps://github.com/pmat0s/pmoscillator/, acessado em $16 / 05 / 2021$.

[16] K. Levenberg, Quart. Appl. Math. 2, 164 (1944).

[17] D. Marquardt, SIAM J. Appl. Math. 11, 431 (1963)

[18] P.R. Gill, W. Murray e M.H. Wright, em: Practical Optimization (Academic Press, London, 1981), p. 136.

[19] B. Gough, GNU scientific library reference manual (Network Theory Ltd. s.l, 2009).

[20] F.M.L de Souza e S.C. Cardoso, Revista Brasileira de Ensino de Física 42, e20200202 (2020).

[21] J.H. Vuolo, Fundamentos da Teoria dos Erros, (Edgard Blücher LTDA, São Paulo, 1996), 2 ed., p. 113. 